A LETTERS JOURNAL EXPLORING THE FRONTIERS OF PHYSICS

\title{
OFFPRINT
}

\section{Echo enabled harmonic generation free electron laser in a mode-locked configuration}

J. R. Henderson and B. W. J. McNeil

EPL, 100 (2012) 64001

Please visit the new website

www.epljournal.org 


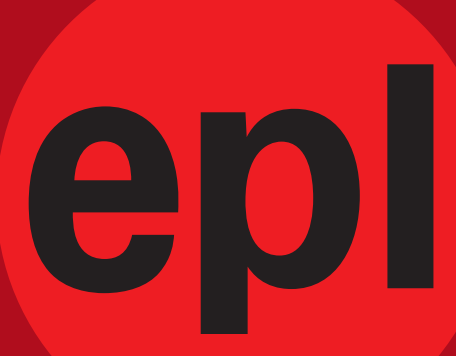

A LetTers JoUrnal EXPLoring the Frontiers of Physics

\section{AN INVITATION TO SUBMIT YOUR WORK}

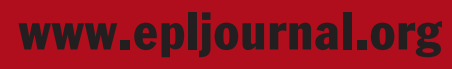

The Editorial Board invites you to submit your letters to EPL

EPL is a leading international journal publishing original, high-quality Letters in all areas of physics, ranging from condensed matter topics and interdisciplinary research

to astrophysics, geophysics, plasma and fusion sciences, including those with application potential.

The high profile of the journal combined with the excellent scientific quality of the articles continue to ensure EPL is an essential resource for its worldwide audience. EPL offers authors global visibility and a great opportunity to share their work with others across the whole of the physics community.

\section{Run by active scientists, for scientists}

EPL is reviewed by scientists for scientists, to serve and support the international scientific community. The Editorial Board is a team of active research scientists with an expert understanding of the needs of both authors and researchers.
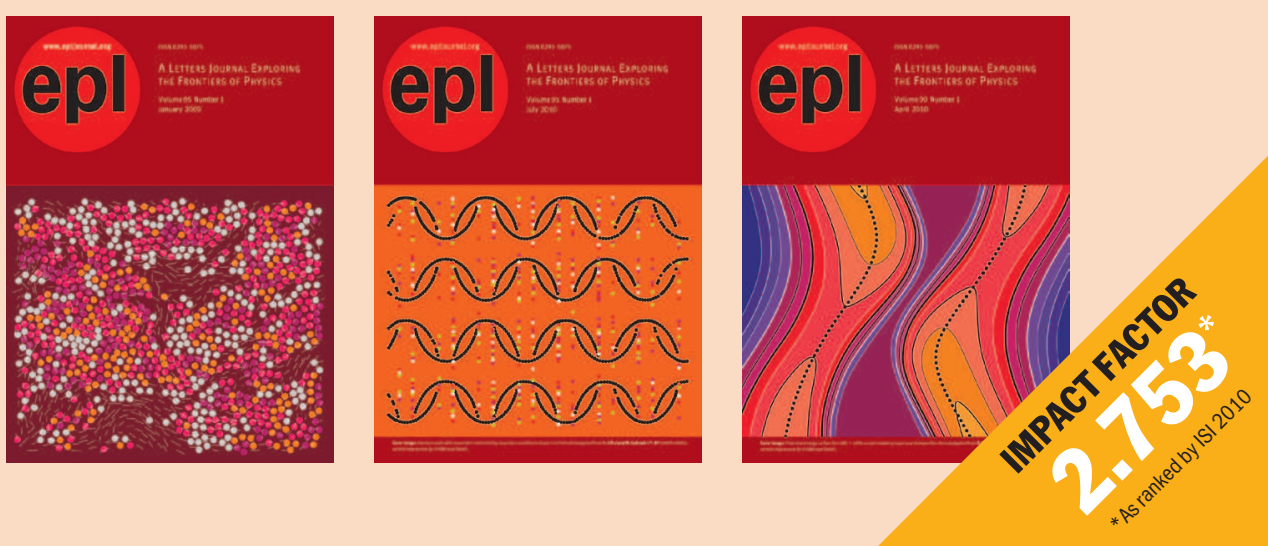

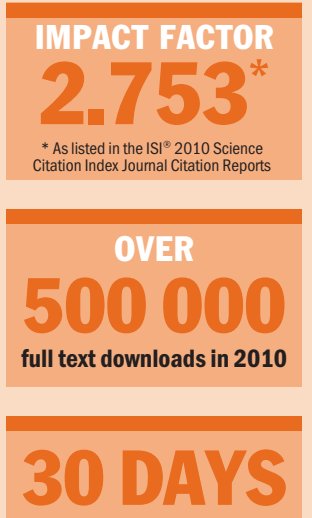

average receipt to online publication in 2010

\section{(1) $0 \longdiv { 6 }$ \\ citations in $\mathbf{2 0 1 0}$ \\ $37 \%$ increase from 2007}

"We've had a very positive experience with EPL, and not only on this occasion. The fact that one can identify an appropriate editor, and the editor is an active scientist in the field, makes a huge difference."

Dr. Ivar Martinv

Los Alamos National Laboratory, USA

\section{Six good reasons to publish with EPL}

We want to work with you to help gain recognition for your high-quality work through worldwide visibility and high citations.

Quality - The 40+ Co-Editors, who are experts in their fields, oversee the entire peer-review process, from selection of the referees to making all final acceptance decisions

Impact Factor - The 2010 Impact Factor is 2.753; your work will be in the right place to be cited by your peers

Speed of processing - We aim to provide you with a quick and efficient service; the median time from acceptance to online publication is 30 days

High visibility - All articles are free to read for 30 days from online publication date

International reach - Over 2,000 institutions have access to EPL, enabling your work to be read by your peers in 100 countries

Open Access - Articles are offered open access for a one-off author payment

Details on preparing, submitting and tracking the progress of your manuscript from submission to acceptance are available on the EPL submission website www.epletters.net.

If you would like further information about our author service or EPL in general, please visit www.epljournal.org or e-mail us at info@epljournal.org.

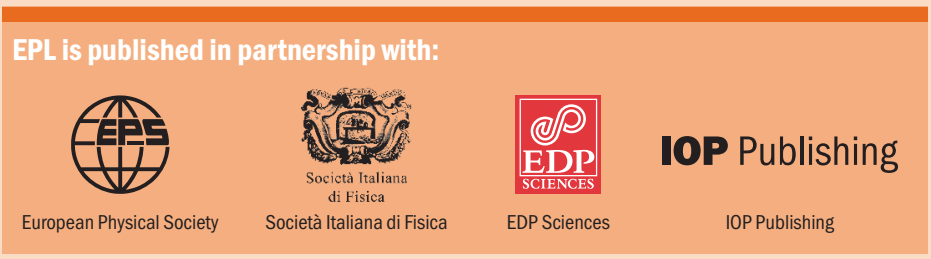

\section{www.epljournal.org}




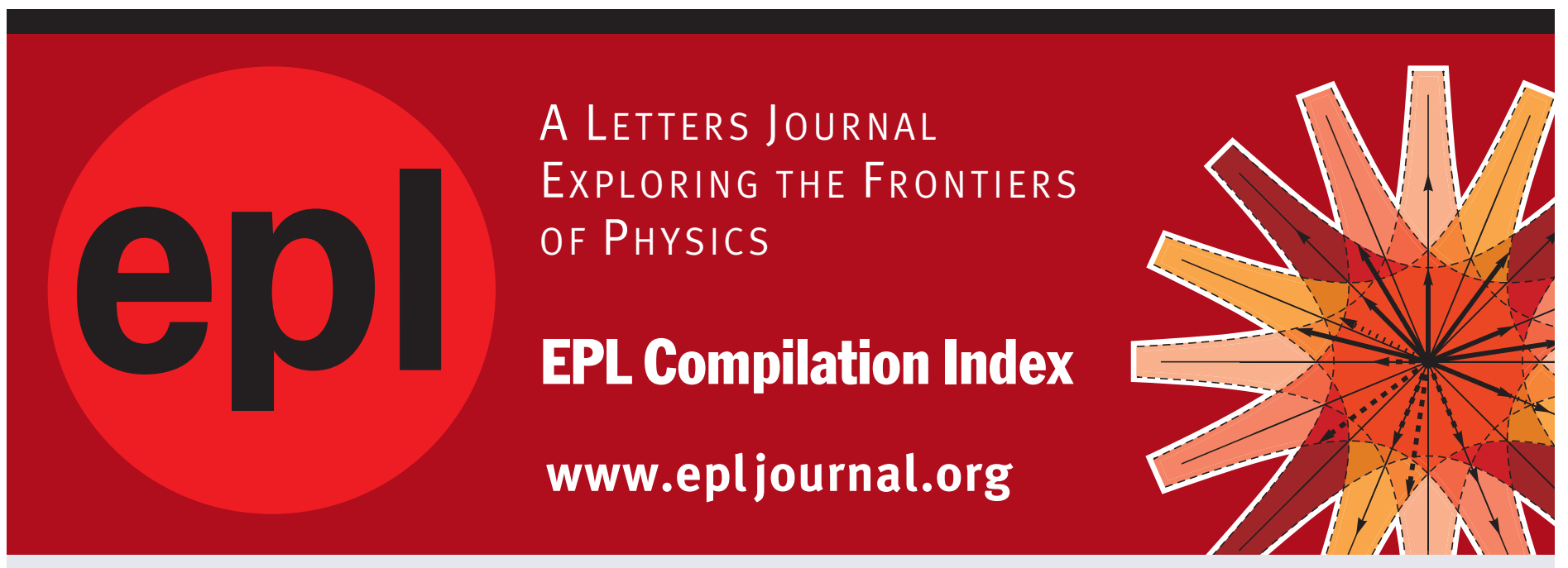

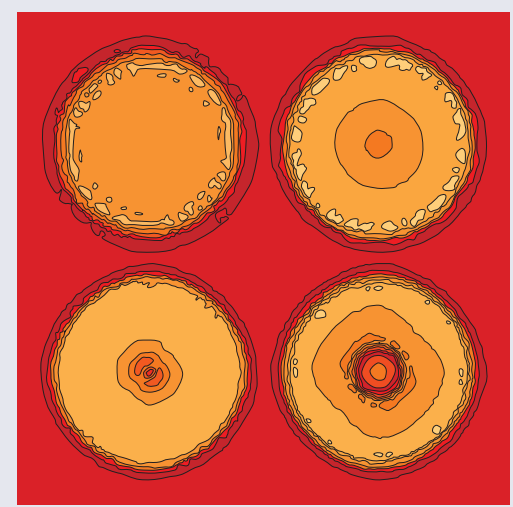

Biaxial strain on lens-shaped quantum rings of different inner radii, adapted from Zhang et al 2008 EPL 8367004.

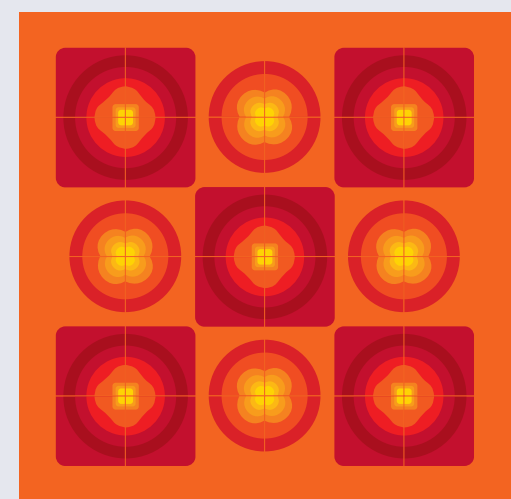

Artistic impression of electrostatic particle-particle interactions in dielectrophoresis, adapted from N Aubry and P Singh 2006 EPL 74623.

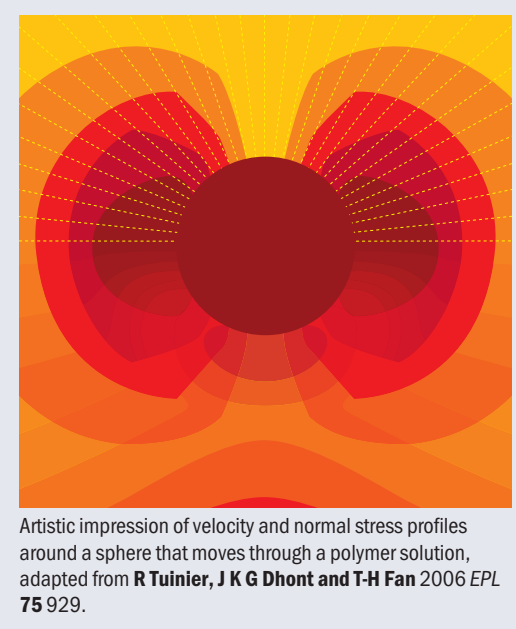

Visit the EPL website to read the latest articles published in cutting-edge fields of research from across the whole of physics.

Each compilation is led by its own Co-Editor, who is a leading scientist in that field, and who is responsible for overseeing the review process, selecting referees and making publication decisions for every manuscript.

- Graphene

- Liquid Crystals

- High Transition Temperature Superconductors

- Quantum Information Processing \& Communication

- Biological \& Soft Matter Physics

- Atomic, Molecular \& Optical Physics

- Bose-Einstein Condensates \& Ultracold Gases

- Metamaterials, Nanostructures \& Magnetic Materials

- Mathematical Methods

- Physics of Gases, Plasmas \& Electric Fields

- High Energy Nuclear Physics

If you are working on research in any of these areas, the Co-Editors would be delighted to receive your submission. Articles should be submitted via the automated manuscript system at www.epletters.net

If you would like further information about our author senvice or EPL in general, please visit www.epljournal.org or e-mail us at info@epljournal.org

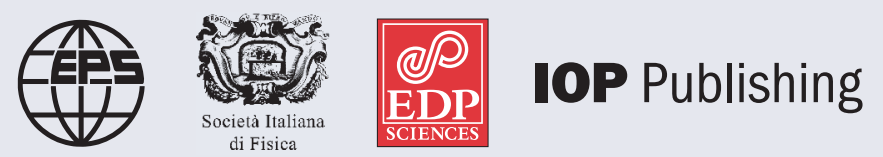

Image: Ornamental multiplication of space-time figures of temperature transformation rules (adapted from T. S. Bíró and P. Ván 2010 EPL 89 30001; artistic impression by Frédérique Swist). 


\title{
Echo enabled harmonic generation free electron laser in a mode-locked configuration
}

\author{
J. R. Henderson ${ }^{1,2}$ and B. W. J. MCNEIL ${ }^{1}$ \\ ${ }^{1}$ SUPA, Department of Physics, University of Strathclyde - Glasgow, G4 ONG, UK, EU \\ ${ }^{2} A S T e C, S T F C$ Daresbury Laboratory and Cockcroft Institute - Warrington, WA4 4AD, UK, EU
}

received 8 October 2012; accepted in final form 27 November 2012

published online 3 January 2013

PACS 41.60.Cr - Free-electron lasers

PACS 42.55.Vc - X- and $\gamma$-ray lasers

PACS 42.65.Re - Ultrafast processes; optical pulse generation and pulse compression

\begin{abstract}
Echo Enabled Harmonic Generation (EEHG) is a method of harmonic up-shifting proposed to extend the temporal coherence properties of Free Electron Lasers (FEL) at shorter wavelengths where coherent laser seed fields are not available. Previous theoretical studies of EEHG have applied periodic boundary conditions to the electron distribution in phase space. It is shown that when these periodic boundary conditions are removed a temporal comb of enhanced electron microbunching is revealed. By matching this comb structure in the electron microbunching to the radiation modes that are generated in a Mode-Locked Optical Klystron (MLOK) FEL configuration, a train of short radiation pulses can be generated.
\end{abstract}

Copyright (c) EPLA, 2012

Introduction. - X-ray Free Electrons Lasers [1] (FEL) are now generating high-brightness, X-ray pulses that are opening up many new areas of science in fields as diverse as the creation of warm dense matter [2], high energy pumping of atoms to population inversion to create an atomic X-ray laser [3] and the making of "molecular movies" of biological processes [4]. The latter processes require femtosecond timescale pulses to investigate transient molecular structure in, e.g. protein nanocrystallography [5] and single virus imaging [6], succinctly described as "dynamic biology" [4].

The general capability of capturing and possibly altering faster electronic processes that guide chemical pathways in the attosecond regime, would help further transform this research area to include imaging of catalysis, graphene, carbon nanotechnology and nanostructure development [7] and the nascent field of quantum biology [8]. This imaging of the faster electronic motion at the quantum level is ultimately the key to a more complete understanding of the functioning of complex molecular and biological systems [9]. There is therefore considerable interest in developing high-brightness, X-ray attosecond duration pulses that would allow the study of these ultra-fast atomic and molecular processes. This letter proposes a method of achieving this by combining the two previously unrelated FEL methods of Echo
Enabled Harmonic Generation (EEHG) [10,11] and the Mode-Locked Optical Klystron (MLOK) [12].

The method of EEHG manipulates electron pulse phase space using two temporally coherent, long wavelength seed lasers and two dispersive chicanes. The electrons are first modulated by a seed laser in an undulator and then dispersed in a chicane. This process is then repeated and a fine microbunching of the electron beam is created at a shorter wavelength while retaining a high level of the temporal coherence from the long wavelength lasers. When propagated through a final radiator undulator, the electron beam emits X-rays at the shorter wavelength of the electron microbunching and with an improved temporal coherence over that generated by self-amplified spontaneous emission which starts from intrinsic shotnoise in the electron beam [13]. Previous models of EEHG have used periodic boundary conditions applied to the electron phase space and simulation codes that average the electron and radiation properties over a radiation wavelength.

In this letter, these limiting assumptions are removed by using the unaveraged FEL simulation code of [14] with the macroparticle loading algorithm of [15]. Using this model, simulations of EEHG up to before the final undulator reveal a frequency comb of modes in the electron microbunching parameter, with adjacent modes 
being separated by the second seed laser frequency. This frequency comb may then be matched to and seed a MLOK undulator-chicane lattice as the final radiator stage of the process to generate a seeded Attosecond Pulse Train (APT).

EEHG pre-radiator stage. - To demonstrate the process, EEHG was simulated with the following parameters for a cold beam with no intrinsic shot-noise. A uniform "flat top" current profile electron pulse was used with electron energy $E_{r}=1.2 \mathrm{GeV}$, charge $Q=100 \mathrm{pC}$ and initial pulse length of $12 \mu \mathrm{m}$ (which is equivalent to a current of $2.5 \mathrm{kA}$ ). The first and second seed laser wavelengths are $\lambda_{1,2}=240 \mathrm{~nm}$ with the final radiating resonant wavelength $\lambda_{r}=10 \mathrm{~nm}$. The electron energy modulation in the first and second modulating stages are $\Delta E_{1}=750 \mathrm{keV}$ $\left(\Delta E_{1} / \rho E_{r}=0.625\right)$ and $\Delta E_{2}=150 \mathrm{keV}\left(\Delta E_{2} / \rho E_{r}=\right.$ $0.125)$ respectively, where the bracketed terms are scaled with respect to the FEL $\rho$-parameter [1] of the final radiator stage. The dispersive strength of the corresponding chicanes are $R_{56}^{(1)}=8.25 \mathrm{~mm}$ and $R_{56}^{(2)}=0.34 \mathrm{~mm}$. The electrons are modelled by macroparticles [15] each assigned a weight $N_{j}$ corresponding to the number of electrons the macroparticle represents. A FEL parameter of $\rho=10^{-3}$ was used as a typical value for the simulations at this resonant wavelength.

Each of the two modulation-dispersion processes of EEHG prior to the radiator stage were simulated by applying an energy modulation to the electron distribution and then dispersing the distribution using the following transformations applied to the initial distribution:

$$
\begin{aligned}
& \gamma_{j}^{(n+1)}=\gamma_{j}^{(n)}+\Delta \gamma_{n+1} \sin \left(z_{j}^{(n)} k_{n+1}\right), \\
& z_{j}^{(n+1)}=z_{j}^{(n)}+R_{56}^{(n+1)}\left[\frac{\gamma_{j}^{(n+1)}-\gamma_{r}}{\gamma_{r}}\right],
\end{aligned}
$$

where $n=0,1$, and bracketed superscripts $(0,1,2)$ referring to the initial conditions, and to the exit from the first and second modulation/dispersive stages, respectively; $\gamma$ is the electron energy in units of the electron rest-mass energy and $\Delta \gamma$ is the energy modulation.

Applying the four transforms of eqs. (1) and (2) develops a microstructure in the electron pulse that contains significant microbunching at higher harmonics of the seed radiation fields used to modulate the electron beam energy.

In demonstrating the principle of EEHG, the work of $[10,11]$ applied periodic boundary conditions in the position of the electrons across a region of the longitudinal $z$-axis. Here, no such boundary conditions are applied and the electron positions are transformed according to (2). The removal of the boundary condition has little effect around the centre of the electron pulse where the dispersive effects are symmetric. The effects of the two modulation and dispersive sections are seen in fig. 1 and result in a phase space that is very similar to that of reported
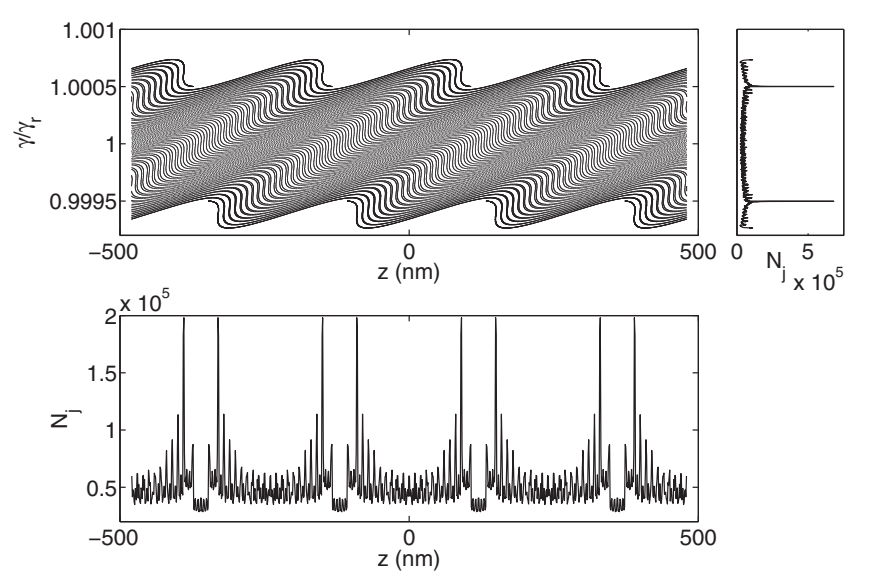

Fig. 1: Electron phase space (top) and histogram of electron numbers (bottom) about the centre of the electron pulse at $z=0$. The particle density is increased for the high and low energy electrons as is indicated by the top right plot.
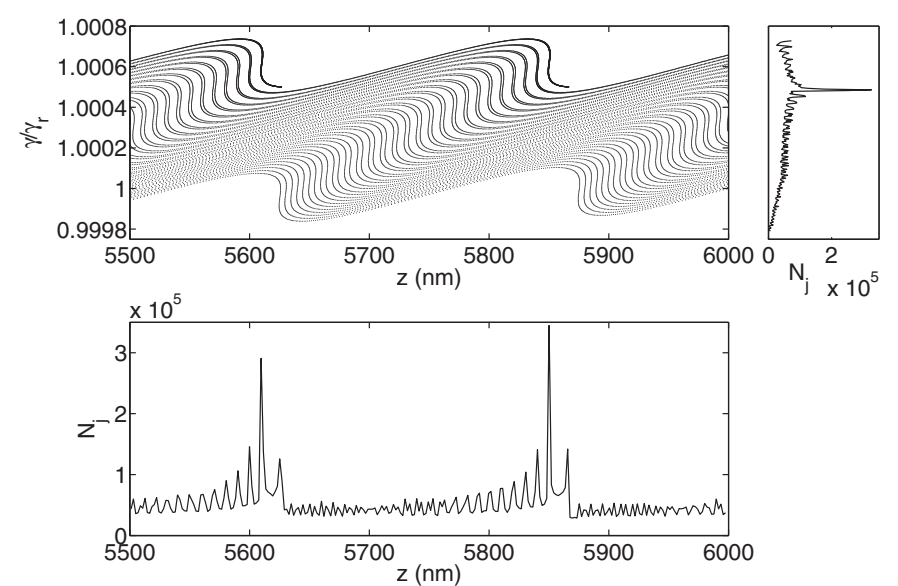

Fig. 2: Electron phase space (top) and histogram of electron numbers (bottom) at the head of the electron pulse. The particle density is increased for the higher energy electrons as is indicated by the top right plot. Single current bands can be seen in the region around $z=5600-5615 \mathrm{~nm}$ with double current bands around $z=5620-5830 \mathrm{~nm}$.

in $[10,11]$. The higher and lower energy electrons, from the extrema of the energy modulated beam, form tight (single) "current bands", whereas electrons close to the initially unmodulated beam energy form a looser (double) current band structure. This is not the case towards the head and tail of the electron pulse where dispersion causes predominantly single current bands formed by the higher and lower energy electrons, respectively. This effect is seen for the case of the head of the electron pulse in fig. 2 .

The underlying process is detailed in fig. 3 which shows the effects of the EEHG process upon the higher energy electrons of the initially modulated beam. The higher energy electrons are seen to form relatively tight energy bands which are then transformed into single current bands around the maxima following the second energy modulation. This creates a series of higher energy current 

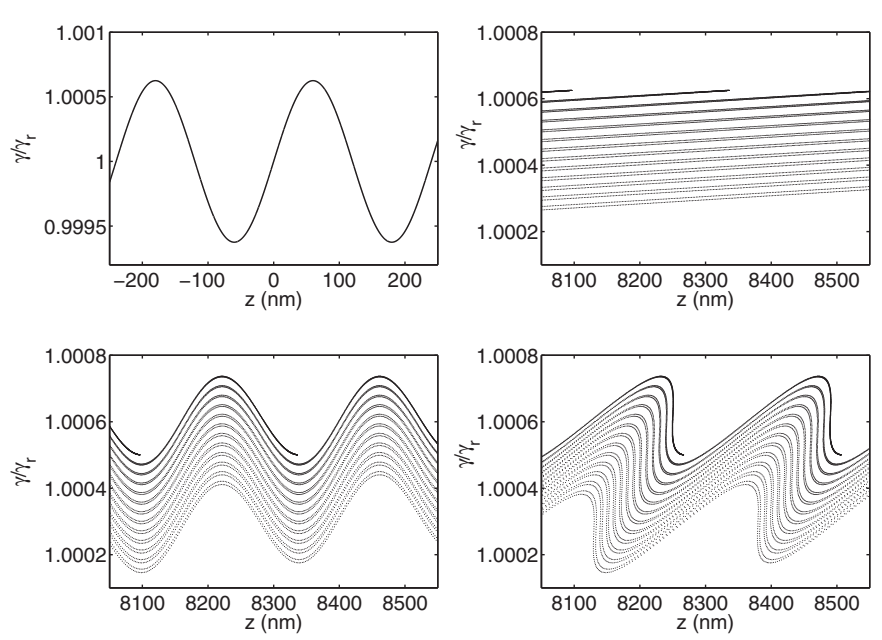

Fig. 3: Both single and double current-bands can be seen to evolve during the pre-radiator EEHG process (left-toright, top-to-bottom): 1) first beam energy modulation, 2) first chicane dispersion, 3) second beam energy modulation, 4) second chicane dispersion. Notice the relatively large shift in the electron positions in $z$ due to the first chicane.

bands at the head of the electron pulse separated by the wavelength of the second seed laser. At the tail of the electron pulse, the EEHG process causes similar single current bands to be formed, but around the minima of the second energy modulation. Thus, dispersion causes the high (low) energy electrons to be dispersed to the head (tail) of the pulse and also a phase difference of $\pi\left(\lambda_{2} / 2\right)$ between the current bands at the head and tail.

A histogram of the full electron number distribution is shown in fig. 4 together with a (unitary) Fourier transform of the electron bunching parameter about the resonant frequency $\omega_{r}$ of the final radiator stage. The Fourier bunching parameter is simply derived from the driving term of the scaled wave equation of [15] and may be written as

$$
b(z, \omega)=\frac{1}{\sqrt{2 \pi}} \frac{1}{n_{p \|}} \sum_{j=1}^{N_{m}} N_{j} e^{-i z\left(k_{r}+k_{u}\right)} e^{i\left(\omega_{r}-\omega\right) t_{j}},
$$

where $n_{p \|}$ is the peak linear electron density, $N_{m}$ is the total number of macroparticles used in the simulation, $k_{u}$ is the undulator wavenumber, $t_{j}=-z_{j} / c \beta_{\|}$is the arrival time of the macroparticle at the undulator entrance at $z=0$ and $\beta_{\|}=v_{z} / c$ is the mean scaled speed of the electron pulse along the undulator $z$-axis. It is seen that a well-defined modal structure is present in the electron bunching parameter with mode separation given of $\Delta \omega / \omega_{r}=10 / 240 \approx 0.042$ corresponding to the seed modulation frequency. The modes are relatively well phasematched as seen from the well-defined peaked periodic microstructure in the electron density histogram.

EEHG radiator. - The pre-bunched electron distribution above was propagated through two different undulator systems, a simple undulator and an undulator-chicane
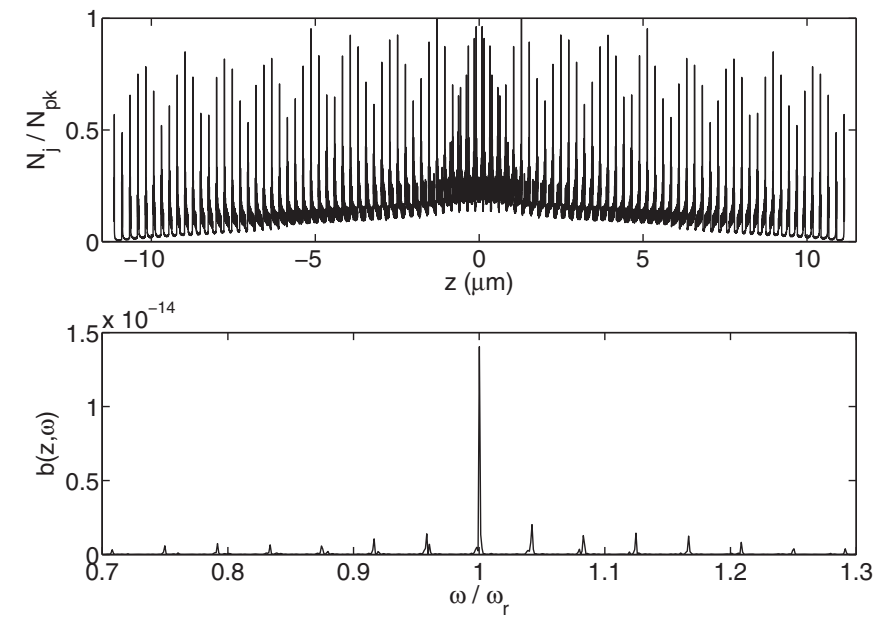

Fig. 4: Histogram of electron numbers (top) normalised with respect to the peak, and the Fourier transform of the bunching parameter $b(z, \omega)$ for the full electron beam distribution showing the modal structure at the end of the EEHG pre-bunching process.

system of the MLOK type, both tuned to the resonant frequency $\omega_{r}$ using the unaveraged simulation code of [14]. The transformations of $(1,2)$ may be rewritten in the universal scaling of [16] as used in the simulations as

$$
\begin{aligned}
& p_{j}^{(n+1)}=p_{j}^{(n)}+\Delta p^{(n+1)} \sin \left(\frac{\bar{z}_{1 j}^{(n)}}{2 \rho h_{n+1}}\right), \\
& \bar{z}_{1 j}^{(n+1)}=\bar{z}_{1 j}^{(n)}+2 \rho D^{(n+1)} p_{j}^{(n+1)},
\end{aligned}
$$

where $\Delta p^{(1,2)}=\Delta \gamma^{(1,2)} / \rho \gamma_{r} ; \quad D^{(1,2)}=k_{r} \rho R_{56}^{(1,2)} ; \quad h_{1,2}=$ $\omega_{r} / \omega_{1,2}$ and $\beta_{\|} \approx 1$ is assumed. At the beginning of the undulator: $\bar{z} \equiv 2 \rho k_{u} z=0 ; \quad \bar{z}_{1 j}=-2 k_{r} \rho c t_{j} \quad$ and $p_{j}=\left(\gamma_{j}-\gamma_{r}\right) / \rho \gamma_{r}$.

Performing the Fourier transform with respect to $\bar{z}_{1}$ defines the scaled frequency as $\bar{\omega}=-\omega / 2 \rho \omega_{r}$ so that

$$
b(\bar{z}, \bar{\omega})=b(z, \omega) \frac{c}{l_{c}} \exp \left(i \frac{\omega z}{c}\right),
$$

where $l_{c}=\lambda_{r} / 4 \pi \rho$ is the cooperation length [16].

Simple undulator. EEHG was first simulated in a simple undulator configuration of scaled length $\bar{z}=1.1$. The scaled radiation and electron pulse parameters are plotted in fig. 5. It is seen that while the radiation generated had some temporal structure, no modal structure is present in Fourier space with emission confined to the resonant frequency. This is consistent with the previous results of $[10,11]$.

MLOK undulator. Here an MLOK undulator-chicane lattice is constructed so that the radiation modes will match the properties of the electron bunching above. Using the notation of [12], each undulator has four periods so that a radiation wavefront will propagate four resonant 

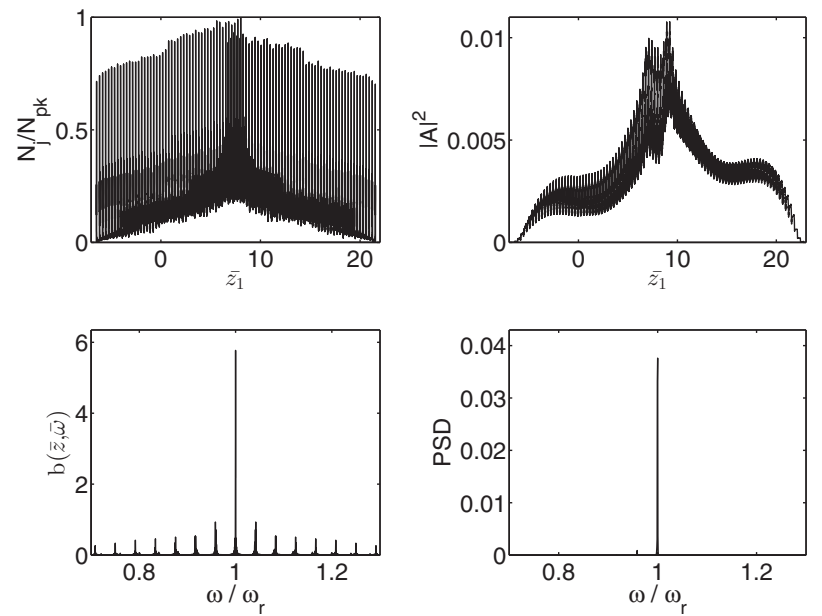

Fig. 5: Electron and radiation pulse at saturation in a simple undulator at $\bar{z} \approx 1.1$ for the normal EEHG case. Plots on the left are: top, normalised electron number histogram (bin size $=$ $\left.\lambda_{r} / 5\right)$; bottom, Fourier transform of bunching $b(\bar{z}, \bar{\omega})$. On the right: top, radiation field amplitude $|A|^{2}$ as a function of $\bar{z}_{1}$; bottom, scaled Power Spectral Density (PSD) showing that emission at resonance dominates.
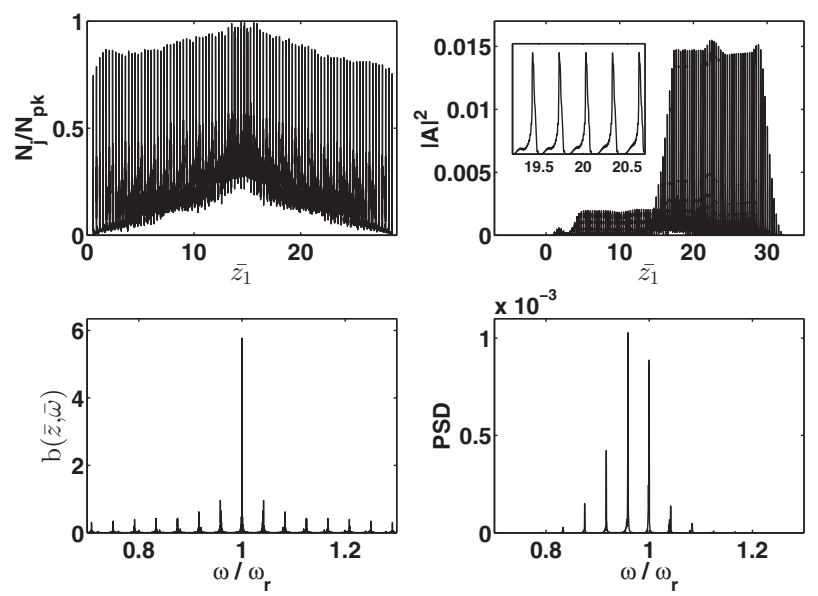

Fig. 6: As fig. 5, but for the MLOK undulator at saturation $(\bar{z} \approx 0.6)$. The inset (top, right) shows more detail expanded in $\bar{z}_{1}$. A well-defined set of phase-matched radiation modes has developed resulting in a train of short radiation pulses. In unscaled units the individual pulse widths are $~ 106$ attoseconds (FWHM) and separated by $\sim 0.8$ femtoseconds corresponding to $\bar{s}=0.3$ in scaled units of $\bar{z}_{1}$.

wavelengths through the electron pulse in each undulator. Each chicane delays the electron pulse by a further twenty wavelengths so that the total slippage of a resonant wavefront in traversing one undulator-chicane module is $s=(4+20) \lambda_{r}=240 \mathrm{~nm}$, which is equal to the second seed laser wavelength, $\lambda_{2}$. In the scaled units of $\bar{z}_{1}$ this corresponds to $\bar{s}=s / l_{c} \approx 0.3$. In this way the relative slippage between radiation and electrons in each undulator-chicane module matches the strong periodic electron microbunching. It is seen from the inset of fig. 6 that this matching generates a periodic train of short radiation pulses (corresponding to full width half-maximum duration of $~ 106$ as) separated by the second seed laser wavelength. This pulse train is equivalent in frequency space to the observed set of modes equally spaced by the frequency of the second seed laser, $\Delta \omega / \omega_{r} \approx 0.042$. It is easily shown from the FEL resonance relation [1] that the frequency range of radiation emission due to the electron energy modulations $\Delta \gamma_{1,2}$ is negligible in comparison with the mode spacing when $\left(\Delta \gamma_{1}+\Delta \gamma_{2}\right) / \gamma_{r} \ll \Delta \omega / 2 \omega_{r}$. Here, this condition is satisfied as $\left(\Delta \gamma_{1}+\Delta \gamma_{2}\right) / \gamma_{r} \approx 4 \times 10^{-2} \Delta \omega / 2 \omega_{r}$. Note that peak powers generated by the higher energy electrons at the head of the radiation pulse train envelope are greater than those generated at the tail by the lower energy electrons. While the higher energy electrons lose energy and fall into resonance to emit strongly, those at lower energies continually fall away from resonance and strong radiation emission. The visibility of radiation pulse train structure is defined as $V=\left(|A|_{\text {max }}^{2}-|A|_{\text {min }}^{2}\right) /\left(|A|_{\text {max }}^{2}+|A|_{\text {min }}^{2}\right)$, were the maximum and minimum values are defined between two adjacent peaks. The effect of introducing an energy spread $\sigma_{E}$ in the initial electron pulse energy decreases the visibility gradually from $V=0.93$ at $1 \mathrm{keV}\left(\sigma_{E} / \rho E_{r}\right.$ $=0.0008)$ to $V=0.78$ at $150 \mathrm{keV}\left(\sigma_{E} / \rho E_{r}=0.125\right)$.

Conclusions. - By removing previous simplifying assumptions in EEHG modelling, a previously unreported temporal structure in the electron pulse microbunching was revealed. This structure can be matched to a modelock FEL (MLOK) configuration which can generate trains of 106 attosecond (FWHM) duration pulses at $10 \mathrm{~nm}$ wavelength. The parameters used here are similar to those used previously to demonstrate EEHG. However, there are no intrinsic reasons why the resonant wavelength and pulse durations cannot be scaled down further into the hard X-ray.

The authors would like to thank LAWREnCE CAMPBELL and David Dunning for helpful discussions. This work received support from STFC Memorandum Of Agreement No. 4163192.

\section{REFERENCES}

[1] McNeil Brian W. J. and Thompson Neil R., Nat. Photon., 4 (2010) 814.

[2] Vinko S. M. et al., Nature, 482 (2012) 59.

[3] Rohringer N. et al., Nature, 481 (2012) 488.

[4] Spence J. C. H., Weierstall U. and Chapman H. N., Rep. Prog. Phys., 75 (2012) 102601.

[5] Chapman H. N. et al., Nature, 470 (2011) 73.

[6] Seibert M. M. et al., Nature, 470 (2011) 78.

[7] Flavell W. R., Proceedings of IPAC2012, New Orleans, U.S.A. (Joint Accelerator Conferences Website (JACoW)) 2012, paper WEI04.

[8] Olaya-Castro A., Nazir A. and Fleming G. R., Philos. Trans. R. Soc. A, 370 (2012) 3613. 
[9] Dixit Gopal, Vendrell Oriol and Santra Robin, Proc. Natl. Acad. Sci. U.S.A., 109 (2012) 1636.

[10] Xiang D. and Stupakov G., Phys. Rev. ST Accel. Beams, 12 (2009) 030702.

[11] Xiang D., Huang Z. and Stupakov G., Phys. Rev. ST Accel. Beams, 12 (2009) 060703.

[12] Thompson N. R. and McNeil B. W. J., Phys. Rev. Lett., 100 (2008) 203901.
[13] Bonifacio R., De Salvo L., Pierini P., Piovella N. and Pellegrini C., Phys. Rev. Lett., 73 (1994) 1.

[14] MeNeil B. W. J., Robi G. R. M. and Jaroszynski D. A., Opt. Commun., 165 (1999) 65.

[15] McNeil B. W. J., Poole M. W. and Robb G. R. M., Phys. Rev. ST Accel. Beams, 6 (2003) 070701.

[16] Bonifacio R., McNeil B. W. J. and Pierini P., Phys. Rev. A, 40 (1989) 4467. 